\title{
Review of: "Efficacy And Renal Safety of Febuxostat In Management of Gout And Chronic Kidney Disease: A Retrospective Study"
}

\author{
Kanon Jatuworapruk ${ }^{1}$
}

1 Thammasat University

Potential competing interests: The author(s) declared that no potential competing interests exist.

This retrospective study attempted to examine the urate-lowering efficacy and renal safety of febuxostat in patients with gout and CKD (stage 2-4). The study subsequently explored factors associated with reaching target serum urate level in the same population.

The manuscript deals with a clinically important subject, but there are some issues that could raise concerns that the current design/methods may not be adequate to fulfill the study's objectives. The followings are major concerns that should be considered when interpreting this study results:

1. There is no control group. The study set out to explore the efficacy of febuxostat, but all patients in the cohort received febuxostat. Consequently, it is difficult to conclude that the outcomes observed were associated with febuxostat. Most previous studies compared patients taking febuxostat with those taking allopurinol and/or placebo. The lack of controls may undermine the validity of the study's conclusion.

2. Study design should be further clarified. The methods section stated that this was a retrospective study. More details are needed.

3. Was the sample size sufficient for a robust regression model?

4. How were eligible patients identified from the EMRS? Were the patients identified using diagnostic codes (ICD-10) or by another method?

5. Logistic regression model requires some clarification:

- How were the variables selected for inclusion into the multivariate logistic regression model? It seemed like a preliminary univariate analysis was used to select final set of variables, but it was not clear how this was performed. From the Table S3, only one variable (acute arthritis) had p-value $<0.05$. However, three other variables (BW, SU and eGFR) were also selected for the multivariate models (Table 3). If another p-value threshold was used for the selection (e.g., p-value $<0.2$ or $<0.1$ ), a different set of variables would have been included in the multivariate model.

- Given the substantial amount of existing literature on this subject, I think variable selection could be done using existing clinical knowledge. One advantage of this selection method is that it would 
ensure that the factors in the final model are clinically meaningful.

- The dependent variable of the logistic regression should be clearly stated in the methods.

- I strongly encourage the authors report the regression coefficient (beta) values for all variables in the regression models, as well as the incept value. These values would indicate the degree and/or manner of association for each predictor.

6. A full list of variables and definition should be provided.

- For example, disease duration could have meant the period between first attack and recruitment date or between first diagnosis and recruitment date.

7. I am particularly interested in the 'acute arthritis' variable. Was this defined as acute arthritis at presentation or at recruitment? The meaning of this variable is unclear, because gout is already defined by the presence of arthritis by the ACR/EULAR-2015 criteria utilized by this study. In that sense, every patient should have already had arthritis at some point during their disease course. The association between 'acute arthritis' and 'achieving target urate' reported in this study is therefore not very meaningful. Further clarification may be needed. 